\title{
OSTEITE FRONTALE POST-SINUSITIQUE ETUDE RÉTROSPECTIVE À PROPOS DE 31 CAS
}

\author{
N. KAFFEL, K. KHAMASSI, Z. SELMI, R. LAHIANI, T. BEN MAKHLOUF, D. NAJEH, M. BEN SALAH, \\ H. HAJRI, M. KHALDI*, M. FERJAOUI \\ SERVICE D'ORL ET DE CHIRURGIE CERVICO-FACIALE. HÔPITAL CHARLES NICOLLE. TUNIS. \\ ${ }^{*}$ SERVICE DE NEUROCHIRURGIE. INSTITUT NATIONAL DE NEUROLOGIE. TUNIS. TUNISIE
}

\begin{abstract}
Objectif : L'ostéite frontale post-sinusitique est définie par l'extension de l'infection du sinus frontal aux structures osseuses adjacentes. Elle constitue une urgence diagnostique et thérapeutique. Le but de ce travail est d'analyser les particularités épidémiologiques, cliniques et paracliniques, et de passer en revue les différentes attitudes thérapeutiques devant cette pathologie.

Matériel et méthodes : II s'agit d'une étude rétrospective portant sur 31 patients diagnostiqués et traités entre 1996 et 2010. Tous les patients ont bénéficié d'un examen ORL et neurologique complet, d'un bilan biologique et d'une tomodensitométrie du massif facial et cérébrale. L’antibiothérapie a été administrée par voie intraveineuse. Le drainage chirurgical du sinus frontal se faisait soit par trépanation, soit par confection d'un volet frontal, soit par mise en place d'un clou de Lemoyne. Le drainage par voie endonasale comportait une méatotomie moyenne avec ethmoïdectomie antérieure. La cranialisation du sinus frontal se faisait par voie coronale. L'évolution a été évaluée sur des critères cliniques, biologiques et radiologiques.

Résultats : L'âge moyen était de 24,4 ans (8-62 ans) et le sex-ratio était de 4,16. Les céphalées frontales (10 cas) et les signes d'hypertension intracrânienne (8 cas) étaient les signes fonctionnels les plus fréquents. Une tuméfaction frontale était notée chez 9 patients, associée dans 5 cas à une tuméfaction orbitaire au niveau de l'angle interne de l'œil. Les signes rhinologiques étaient dominés par la rhinorrhée purulente (9 cas) et l'obstruction nasale (5 cas). L'endoscopie nasale trouvait du pus au niveau du méat moyen dans 12 cas.

La TDM a révélé un aspect typique d'ostéite frontale sous forme d'un foyer d'ostéolyse. Un abcès sous-périosté était associé chez 7 patients. Une extension intracrânienne a été notée dans 15 cas et orbitaire dans 7 cas. L'examen bactériologique a mis en évidence un germe dans 8 cas. Les germes les plus fréquents étaient le streptocoque et le staphylocoque aureus ( 3 cas pour chacun).

Le traitement antibiotique a été instauré d'emblée chez tous les patients. Pour le traitement chirurgical initial, 9 patients ont bénéficié d'un drainage frontal et 2 autres d'un drainage orbitaire. Par ailleurs, 4 patients ont eu un drainage d'abcès sous périosté seul. Celui-ci a été associé à une cranialisation d'emblée dans un autre cas. Pour les patients ayant des complications endocrâniennes, un drainage d'empyème a été réalisé dans 14 cas, associé chez 6 d'entre eux à une cranialisation d'emblée. Un drainage d'abcès cérébral a été pratiqué chez un autre patient.

L'évolution clinique et radiologique a été favorable chez 24 patients $(77,4 \%)$. Les sept autres patients ont nécessité une reprise chirurgicale devant la persistance ou l'aggravation de la symptomatologie clinique. L'évolution ultérieure a été favorable.

Conclusion : L'ostéite frontale post-sinusitique est une affection rare et grave. Son diagnostic, qui repose sur la clinique et l'imagerie, doit être précoce. Un traitement adéquat doit être instauré afin de prévenir des complications qui peuvent mettre en jeu le pronostic vital.
\end{abstract}

Mots-clés : ostéite, sinus frontal, sinusite, tomodensitométrie, drainage, cranialisation

\section{SUMMARY}

Objective : Post-sinusitic frontal osteitis is defined as an extension of frontal sinus infection to the adjacent bony structures. It is an emergency that should rapidly be diagnosed and treated. The aim of this study is to analyze epidemiologic, clinical and paraclinical features, and to review different therapeutic modalities of this disease.

Materials and methods : We carry a retrospective study about 31 patients diagnosed and treated between 1996 and 2010. All patients underwent complete ENT and neurological examination, biological investigations, sino-nasal and cerebral CT scan. Antibiotherapy was administrated intravenously. Surgical drainage of the frontal sinus was performed either by trephination, by osteoplastic flap confection, or by Lemoyne drain placement. Endonasal drainage consisted of middle meatotomy with anterior ethmoidectomy. Frontal sinus cranialisation was performed with coronal approach. Evolution was assessed on clinical, biological and radiological criteria. 
Results : Mean age was 24,4 years (8-62 years) and sex-ratio 4,16. Frontal headache (10 cases) and intracranial hypertension signs (8 cases) were the most frequent functional symptoms. Frontal tumefaction was noted in 9 patients, associated in 5 cases with orbital tumefaction of the internal eye angle. Rhinological signs were essentially purulent rhinorrhea (9 cases) and nasal obstruction (5 cases). Nasal endoscopy showed purulent secretions at the middle meatus in 12 cases.

CT scan revealed a typical aspect of frontal osteitis with bony lysis. A sub-periostal abscess was associated in 7 patients. Intracranial extension was noted in 15 cases and orbital extension in 7 cases. Bacteriological examination was positive in 8 cases. Most frequent bacteria were streptococcus and staphylococcus aureus (3 cases each).

Antibiotic therapy was initially administrated in all cases. Concerning initial surgical treatment, 9 patients underwent frontal drainage and 2 others orbital drainage. On the other hand, 4 patients underwent only sub-periostal abscess drainage. This one was associated with cranialisation in another case. For patients having endocranial complications, empyema drainage was realized in 14 cases, associed in 6 of them with cranialisation. Cerebral abscess drainage was performed in one other patient. Clinical and radiological evolution was favourable in 24 patients $(77,4 \%)$. The seven other patients were reoperated because of persistence or aggravation of clinical symptoms. Later evolution was favourable.

Conclusion : Post-sinusitic frontal osteitis a rare and serious affection. Diagnosis, based on clinical and radiological features, should be early made. Adequate treatment have to be instituted to prevent life-threatening complications.

Keywords : osteitis, frontal sinus, sinusitis, computed tomography, drainage, cranialisation

\section{INTRODUCTION}

L'ostéite frontale post-sinusitique, appelée également ostéomyélite par les anglo-saxons, est définie par l'extension de l'infection du sinus frontal aux structures osseuses adjacentes. C'est une affection devenue rare de nos jours étant donné l'efficacité des antibiotiques dans le traitement des sinusites (1). Elle constitue une urgence diagnostique et thérapeutique afin d'éviter une atteinte osseuse extensive et destructrice pouvant être responsable de complications endocrâniennes.

Le but de ce travail est d'analyser les particularités épidémiologiques, cliniques et paracliniques, et de passer en revue les différentes attitudes thérapeutiques devant cette pathologie.

\section{MATÉRIEL ET MÉTHODES}

II s'agit d'une étude rétrospective portant sur 31 patients diagnostiqués et traités entre 1996 et 2010 . Ces patients ont été pris conjointement par le service d'ORL de l'Hôpital Charles Nicolle et le service de neurochirurgie de I'Institut National de Neurologie de Tunis.

Tous les patients ont bénéficié d'un examen ORL et neurologique complet, d'un bilan biologique et d'une tomodensitométrie du massif facial et cérébrale. L'antibiothérapie a été administrée par voie parentérale. Elle était basée sur l'association d'une céphalosporine de troisième génération ou d'une fluoroquinolone avec un aminoside et le métronidazole ou avec un anti-staphylococcique (fosfomycine, vancomycine ou teïcoplanine). Sa durée variait entre 4 et 8 semaines. Le drainage chirurgical du sinus frontal se faisait soit par trépanation, soit par confection d'un volet frontal, soit par mise en place d'un clou de Lemoyne. Le drainage par voie endonasale a été réalisé chez 2 patients. II comportait une méatotomie moyenne avec ethmoïdectomie antérieure (drainage type
I de Draf). La cranialisation du sinus frontal se faisait par voie coronale, avec taille d'un volet frontal, nettoyage des foyers ostéitiques, effondrement de la paroi postérieure des sinus et mise en place de taquets osseux et musculaires pour imperméabiliser le canal naso-frontal.

L'évolution a été évaluée sur des critères cliniques, biologiques et radiologiques.

\section{RESULTATS}

L'âge moyen était de 24,4 ans (8-62 ans). Quatre-vingts pourcent des patients étaient âgés de moins de 30 ans. Le sex-ratio était de 4,16. Quatre patients avaient des antécédents de traumatisme crânien ou du massif facial (dont 2 cas de fractures de l'os frontal). Deux patients avaient un diabète type 1 .

Le délai moyen de consultation était de 17 jours (5-30 jours). Les signes neurologiques étaient le motif de consultation le plus fréquent. Ils étaient représentés par les céphalées frontales isolées (10 cas), les signes d'hypertension intracrânienne avec céphalées et vomissements en jet (8 cas), des crises comitiales tonico-cloniques généralisées (5 cas), des signes de localisation avec hémiparésie ( 3 cas) et des troubles de la conscience $(2$ cas). Une tuméfaction frontale était notée chez 9 patients, associée dans 5 cas à une tuméfaction orbitaire au niveau de l'angle interne de l'œil. Un patient avait une diminution associée de l'acuité visuelle. Les signes rhinologiques étaient dominés par la rhinorrhée purulente (9 cas) et l'obstruction nasale (5 cas). Par ailleurs, 14 patients rapportaient la notion de fièvre prolongée.

L'endoscopie nasale trouvait du pus au niveau du méat moyen dans 12 cas et un polype nasal chez un patient. Neuf patients avaient une tuméfaction frontale inflammatoire, associée à une fistule laissant sourdre du pus dans 3 cas. L'examen ophtalmologique a montré un œdème 
palpébral (9 cas), une tuméfaction de l'angle interne de l'œil (5 cas) ou une exophtalmie (5 cas). Par ailleurs, un œdème papillaire a été noté dans 4 cas et un syndrome méningé chez 3 patients.

Les données de l'examen physique sont résumées dans le tableau I.

\begin{tabular}{|l|c|}
\hline \multicolumn{1}{|c|}{ Examen physique } & Nombre de cas \\
\hline Rhinologique & 12 \\
pus au méat moyen & 4 \\
polype nasal & \\
\hline Face & \\
tuméfaction frontale & 9 \\
fistule purulente frontale & 3 \\
\hline Ophtalmologique & \\
œedème palpébral & \\
tuméfaction de l'angle interne de l'œil & 5 \\
exophtalmie & 5 \\
diminution de l'oculomotricité & 2 \\
fistule purulente orbitaire & 1 \\
ptosis & 1 \\
chémosis & 1 \\
\hline Neurologique & 3 \\
œedème papillaire & 3 \\
syndrome méningé & \\
hémiparésie & \\
troubles de conscience & \\
\hline
\end{tabular}

\section{Tableau I: Données de l'examen physique}

La tomodensitométrie du massif facial a révélé un aspect typique d'ostéite frontale sous forme d'un foyer d'ostéolyse (Figures 1, 2). La table interne était atteinte dans 16 cas, la table externe dans 5 cas et les deux tables dans 2 cas. La multiplicité des foyers ostéitiques a été notée dans 9 cas. Un séquestre osseux a été noté dans un cas (Figure 3). Par ailleurs, un comblement fronto-ethmoïdal total ou partiel a été noté chez tous les patients. Un abcès sous-périosté ou «Pott's Puffy tumor» a été noté chez 7 patients (Figure 4).

La TDM cérébrale a révélé un empyème sous-dural chez 8 patients, un empyème extra-dural chez 6 patients et un abcès cérébral dans un cas. Les complications orbitaires étaient dominées par la cellulite préseptale (2 cas), rétroseptale ( 2 cas), l'abcès sous-périosté ( 1 cas) et l'abcès orbitaire ( 2 cas). Une thrombophlébite du sinus caverneux était notée chez un patient.

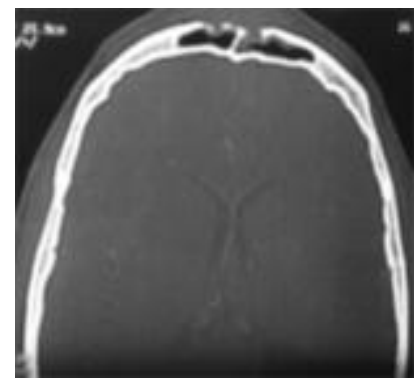

Figure 1 (TDM, coupe axiale) :

Lyse de la paroi antérieure des 2 sinus frontaux

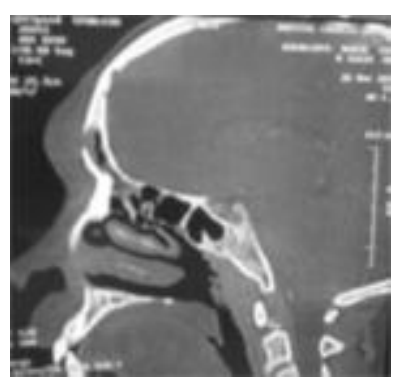

Figure 2 (TDM, reconstruction sagittale) : Lyse de la paroi antérieure du sinus frontal

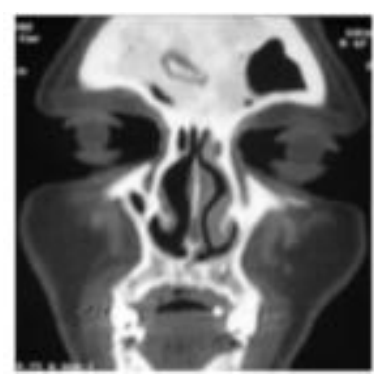

Figure 3 (TDM coupe coronale) :

Séquestre osseux au niveau du sinus frontal droit

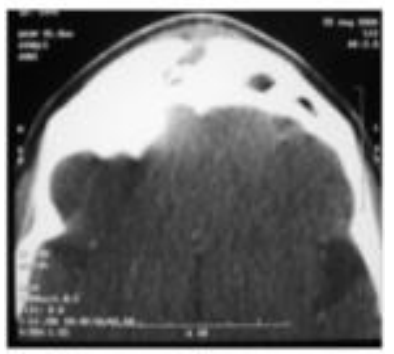

Figure 4 (TDM, coupe axiale) :

Abcès sous-périosté (Pott's Puffy tumor)

La numération formule sanguine a montré une hyperleucocytose chez 26 patients. Un prélèvement de pus a été réalisé chez 18 patients. L'examen bactériologique a mis en évidence un germe dans 8 cas. Les germes les plus 
fréquents étaient le streptocoque et le staphylocoque aureus ( 3 cas pour chacun). Un patient était infecté par le pseudomonas et un autre par l'entérobacter.

La prise en charge avait pour but de traiter la sinusite, d'éradiquer l'ostéite et les autres complications associées. II comportait un volet médical basé sur une antibiothérapie probabiliste adaptée secondairement aux données de l'antibiogramme, et un volet chirurgical faisant appel au drainage associée ou non à une cranialisation du sinus frontal. Le traitement médical a été indiqué d'emblée chez tous les patients. Le traitement chirurgical est détaillé dans le tableau II.

Ainsi, 9 patients ont bénéficié d'un drainage frontal, et 2 patients d'un drainage orbitaire. Quatre patients ont eu un drainage d'abcès sous périosté seul. Celui-ci a été associé à une cranialisation d'emblée chez un autre patient. Pour les patients ayant des complications endocrâniennes, un drainage d'empyème a été réalisé dans 14 cas, associé chez 6 d'entre eux à une cranialisation d'emblée. Un drainage d'abcès cérébral a été pratiqué chez un autre patient.

\begin{tabular}{|l|c|}
\hline \multicolumn{1}{|c|}{ Traitement chirurgical initial } & Nombre de cas \\
\hline Drainage frontal & 4 \\
trépanation & 2 \\
volet frontal & 2 \\
par la perte de substance osseuse spontanée & 1 \\
clou de Lemoyne & 2 \\
Drainage d'abcès orbitaire & 4 \\
Drainage d'abcès sous-périosté & 1 \\
Drainage d'abcès sous-périosté + cranialisation & 8 \\
Drainage d'empyème \pm d'abcès sous-périosté & 6 \\
Drainage d'empyème + cranialisation & 1 \\
Drainage d'abcès cérébral & \\
\hline
\end{tabular}

Tableau II: Traitement chirurgical initial

L'évolution clinique et radiologique a été favorable chez 24 patients $(77,4 \%)$. Deux patients, ayant bénéficié d'une trépanation initiale, ont présenté respectivement une fistule purulente persistante et des signes neurologiques, nécessitant un drainage par volet frontal pour le premier et une cranialisation pour le deuxième. Un patient traité initialement par volet frontal a nécessité une cranialisation devant la persistance de fistule purulente. Chez quatre patients ayant bénéficié initialement d'un drainage d'empyème, on a noté une persistance ou une aggravation des signes neurologiques, en rapport avec une récidive ou une persistance de l'empyème (3 cas) ou l'apparition d'une encéphalite associée à une thrombophlébite cérébrale (1 cas). Ces quatre patients ont été repris chirurgi- calement et ont bénéficié d'une cranialisation secondaire, avec bonne évolution (régression de la symptomatologie fonctionnelle initiale).

\section{DISCUSSION}

L'ostéite frontale est une complication grave de la sinusite frontale. C'est une affection devenue rare au cours de ces dernières décennies grâce à l'antibiothérapie $(2,3)$. La gravité de cette pathologie est tributaire de son évolution quasi inéluctable, en l'absence de prise en charge thérapeutique correcte, vers des complications endocrâniennes qui risquent de compromettre le pronostic vital. L'obstruction du canal naso-frontal est l'élément clé à partir duquel s'édifie l'infection sinusienne et par la suite l'ostéite frontale. Cette entrave au drainage du sinus frontal entraîne une hypoxie et favorise la prolifération microbienne, induisant une réponse immunitaire non spécifique qui va aggraver l'hypoxie par l'intermédiaire de l'inflammation de la muqueuse. La sinusite frontale ainsi constituée et non drainée, va s'étendre à l'os adjacent créant des foyers ostéitiques et des embols veineux septiques. Cette propagation par la circulation veineuse est le mécanisme physiopathologique qui explique l'ostéomyélite des os du crâne et certaines complications intracrâniennes et orbitaires.

L'ostéite frontale post-sinusitique est essentiellement l'apanage de l'adulte. Elles sont rares chez l'enfant car la pneumatisation du sinus frontal ne commence qu'à partir de l'âge de 6 ans. Il existe une légère prédominance masculine (4). Elle survient aussi bien au cours des sinusites frontales aiguës qu'au cours des poussées de réchauffement des sinusites chroniques avec une répartition égale (2). Plusieurs facteurs augmentant le risque de sa survenue sont retrouvés dans la littérature: le traumatisme crânien, l'immuno-dépression, les antécédents de chirurgie des sinus, les déhiscences congénitales ou acquises de la table interne du sinus frontal.

L'ostéite frontale peut revêtir plusieurs aspects cliniques. La forme aiguë est celle d'une sinusite aiguë qui évolue depuis moins de 3 semaines, avec un syndrome infectieux sévère. Les céphalées frontales associées sont tenaces et rebelles. L'examen trouve, outre la fièvre élevée, un œdème frontal douloureux localisé au-dessous de l'angle interne de l'œil. Parfois, il s'agit d'une véritable tuméfaction fluctuante ou «Pott's puffy tumor » des auteurs anglo-saxons et qui représente un signe révélateur d'une ostéite de la table externe (5). Cette forme clinique a été retrouvée chez 7 de nos patients. L'examen des fosses nasales trouve habituellement du pus au niveau du moyen moyen $(3,6)$.

L'ostéite peut également se manifester sous une forme subaiguë, faisant suite à une sinusite aiguë mal ou insuffisamment traitée. La reprise de la fièvre et l'apparition de signes locaux à type d'œdème frontal et de douleur sont évocateurs. Un mode plus torpide se voit surtout chez le 
diabétique, l'immunodéprimé ou le sujet ayant des antécédents de radiothérapie (7).

Les formes révélées par une complication sont rares (8). Elles se manifestent soit par un syndrome neurologique en rapport avec une complication endocrânienne, soit par des signes ophtalmologiques en rapport avec une complication oculo-orbitaire. Les signes neurologiques sont dominés par les céphalées, les signes d'hypertension intracrânienne ou de localisation et le syndrome méningé. Plus rarement, il s'agit de crises comitiales ou de coma. Les signes ophtalmologiques sont représentés par la tuméfaction orbitaire, l'exophtalmie, l'œdème palpébral, le ptosis, le chémosis, voire l'ophtalmoplégie et la cécité. Dans notre série, l'examen physique révélait des signes ophtalmologiques dans $77,4 \%$ et des signes neurologiques dans $38,7 \%$ des cas.

La TDM du massif facial et cérébrale en fenêtre osseuse constitue l'examen clé pour diagnostiquer une ostéite frontale post-sinusitique et rechercher une complication endocrânienne. Elle montre des zones hypodenses caractéristiques de lyse osseuse au niveau des parois sinusiennes antérieure et postérieure, associées à des éventuelles fistules sous forme de pertuis osseux (9). L'IRM montre un hypersignal localisé en séquence T1 contrastant avec l'aspect sombre de l'os normal. De plus, elle permet de mieux évaluer l'atteinte orbitaire et endocrânienne. La scintigraphie au technétium 99 montre les lésions osseuses plus précocement que l'imagerie par rayons $X$. Celle au gallium 67 est surtout indiquée dans le cadre de la surveillance post-thérapeutique.

L'examen bactériologique avec antibiogramme est indispensable. Les germes les plus incriminés dans l'ostéite frontale sont le staphylocoque doré, le streptocoque viridans et les anaérobies (10). L'infection est souvent polymicrobienne. L'atteinte fongique est exceptionnelle (11). Le but du traitement est d'éradiquer l'ostéite, de traiter l'infection sinusienne originelle et les éventuelles complications associées. Le traitement médical est indispensable et est basé sur une antibiothérapie probabiliste débutée dès les premières heures d'hospitalisation. Elle vise essentiellement les germes gram positif et les anaérobies. Les céphalosporines de troisième génération, les fluoroquinolones, les aminosides, le métronidazole et antistaphylococciques (fosfomycine, vancomycine, teïcoplanine) sont les molécules les plus utilisées. Cette antibiothérapie sera adaptée en fonction de l'antibiogramme, et administrée par voie parentérale pendant 3 semaines puis par voie orale. La durée totale du traitement varie entre 4 et 8 semaines. La normalisation de la scintigraphie au gallium représente le meilleur critère objectif de guérison permettant l'arrêt de l'antibiothérapie.

Le traitement chirurgical fait appel à des différentes techniques. La trépanation du sinus frontal se fait soit avec pose d'un clou de Lemoyne, soit par trépanation à minima à la fraise. Elle permet le drainage et le lavage du sinus frontal et est indiquée dans les sinusites bloquées. Le drainage chirurgical est réalisé par voie fronto-sourcilière communément appelée voie de Jacques. Le volet osseux confectionné permet de contrôler les lésions endosinusiennes et la paroi postérieure.

Dans le cadre de l'ostéite frontale post-sinusitique, les techniques de trépanation et de drainage chirurgical sont indiquées dans les ostéites minimes de la table externe. Dans notre série, le drainage frontal a été réalisé initialement chez 9 patients (29\% des cas). Actuellement, les techniques de drainage frontal par voie endoscopique sont de plus en plus réalisées $(12,13,14,15)$. Ces techniques donnent des résultats comparables aux techniques classiques et ont l'avantage d'être moins invasives (16).

Si les techniques précédentes de drainage sont mises en échec ou si l'ostéite est étendue ou atteint la table interne, il convient alors d'envisager des techniques d'exclusion du sinus frontal $(17,18)$. Deux approches différentes sont possibles:

- Le comblement du sinus frontal pour les ostéites étendues de la table externe: après abord coronal, on taille un volet osseux aux dépens de la paroi antérieure en réséquant le foyer ostéitique, puis on enlève soigneusement toute la muqueuse. Le choix du tissu de comblement est variable : muscle temporal, graisse abdominale, tissu osseux prélevé au niveau de la crête iliaque. Les résultats ne sont pas modifiés par le type de matériel de comblement utilisé, mais par la qualité de l'exérèse de la muqueuse sinusienne et de la fermeture de l'ostium nasofrontal (19).

Certains auteurs réalisent ce comblement sous contrôle endoscopique après abord par voie fronto-sourcilière (20). Il s'agit d'une approche moins invasive et aussi efficace que l'approche classique (20).

- L'exclusion neurochirurgicale (cranialisation) du sinus frontal (21): après abord coronal et taille de volet osseux, on réalise une ablation de la paroi postérieure du sinus, un curetage soigneux de toute la muqueuse et une obturation du canal naso-frontal. Cette technique est indiquée dans les ostéites de la table interne et pour l'évacuation d'une collection endocrânienne associée $(21,22,23)$. Dans notre série, cette technique a été pratiquée d'emblée chez 7 patients. Elle a été réalisée secondairement chez 6 des 7 patients qui ont eu une évolution défavorable après traitement initial.

Qu'il s'agisse de cranialisation ou de comblement, l'ablation du volet frontal antérieur ostéitique va causer un préjudice esthétique. De plus, dans la cranialisation, elle va mettre l'endocrâne en communication directe avec la peau. Une cranioplastie doit ainsi être envisagée. Elle fait appel à l'hydroxyapatite, aux greffons osseux autologues, au ciment acrylique (Palacos) ou au titanium (24).

L'évolution favorable est jugée sur des critères cliniques (disparition de la fièvre et des céphalées), radiologiques 
(absence d'hyperfixation à la scintigraphie au gallium, régression du foyer ostéitique au scanner) et biologiques (disparition du syndrome inflammatoire biologique).

\section{CONCLUSION}

L'ostéite frontale post-sinusitique est une affection rare est grave. Son diagnostic, qui repose sur la clinique et l'imagerie, doit être précoce. Un traitement adéquat doit être instauré afin de prévenir des complications qui peuvent mettre en jeu le pronostic vital. La prévention repose essentiellement sur le traitement correct de chaque sinusite frontale.

\section{REFERENCES}

1) Prasad KC, Prasad SC, Mouli N, Agarwal S. Osteomyelitis in the head and neck. Acta Otolaryngol 2007;127:194-205.

(2) Goldberg A, Ororlan G, Anderson T. Complications of frontal sinusitis and their management. Otolaryngol Clin North Am 2001;34:211-25.

(3) Baru R, Todor R, Kassof S. Pott's Puffy Tumor: The forgotten entity. J Neurosurg 1996;84:110-2.

(4) Clayman G, Adams G, Paugh D, Koopmann C. Intracranial complications of paranasal sinusitis: a combined institutional review. Laryngoscope 1991;101:234-9.

(5) Masterson L, Leong P. Pott's puffy tumour: a forgotten complication of frontal sinus disease. Oral Maxillofac Surg 2009;13:115-7.

(6) Banooni P, Rickman L, Ward D. Pott's puffy tumor associated with intracranial methamphetamine. JAMA 2000;283:1293.

(7) Daya S. A silent intracranial complication of frontal sinusitis. J Laryngol Otol 1990;104:645-7.

(8) Younis RT, Lazar RM, Anand VR. Intracranial complications of sinusitis: a 15year review of 39 cases. Ear Nose Throat J 2002;81:636-44.

(9) Milo R, Schiffer J, Karpuch J, Sarfaty S, Shikar S. Frontal bone osteomyelitis complicating frontal sinusitis caused by Haemophilus influenza type A. Rhinology 1991;29:151-3.

(10) Maccabee M, Hwang P. Medical therapy of acute and chronic frontal rhinosinusitis. Otolaryngol Clin North Am 2001;34:41-57.

(11) Effat KG, Karam M, El-Kabani A. Pott's puffy tumour caused by mucormycosis. J Laryngol Otol 2005:119:643-5.

(12) Landsberg R, Friedman M. A minimally invasive endoscopic approach to chronic isolated frontal sinusitis. Oper Tech Otolaryngol 2006;17:184-8.

(13) Weber R, Draf W, Kratzsch B, Hosemann W, Schaefer SD. Modern concepts of frontal sinus surgery. Laryngoscope 2001;111:137-46.

(14) Pételle B, Sauvaget E, Kici S, Tran Ba Huy P, Herman P. Résection endo- scopique du plancher du sinus frontal selon la technique de Draf. Résultats à long terme et algorithme thérapeutique. Ann Otolaryngol Chir Cervicofac 2003;120:14-20

(15) Landsberg R, Segev Y, Friedman M, Flis DM, Derowe A. A targeted endoscopic approach to chronic isolated frontal sinusitis. Otolaryngol Head Neck Surg 2006;134:28-32.

(16) Catalano P, Roffman E. Outcome in patients with chronic sinusitis after the minimally invasive sinus technique. Am J Rhinol 2003;17:17-22.

(17) Lee JJ, Jacobs JB. Frontal sinusitis. Oper Tech Otolaryngol Head Neck Surg 2001;12:80-2.

(18) Soyka MB, Annen A, Holzmann D. Where endoscopy fails: indications and experience with the frontal sinus fat obliteration. Rhinology 2009;47:136-40. (19) Weber R, Draf W, Keerl R, et al. Magnetic resonance imaging following fat obliteration of the frontal sinus. Neuroradiology 2002;44:52-8.

(20) Ung F, Sindwani R, Metson R. Endoscopic frontal sinus Oobliteration: A new technique for the treatment of chronic frontal sinusitis. Otolaryngol Head Neck Surg 2005;133:551-5.

(21) Ameline E, Wagner I, Delbove H, Coquille F, Visot A, Chabolle F. La cranialisation des sinus frontaux. A propos de 19 cas. Ann Otolaryngol Chir Cervicofac 2001;118:352-8.

(22) Constantinidis J, Weber R, Brune M, Draf W, Iro M. Cranialisation of the frontal sinus: Indications, technique and results. HNO 2000;48:361-6.

(23) Altman KW, Austin MB, Tom LWC, Knox GW. Complications of frontal sinusitis in adolescents: case presentations and treatment options. Int J Pediatr Otorhinolaryngol 1997;41:9-20.

(24) Bücheler M, Weihe S, Eufinger H, Wehmöller M, Bootz F. Reconstruction of the frontal bone with individual titanium implants after surgical therapy of osteomyelitis of the frontal bone. HNO 2002;50:339-46. 